LMU-07/94

PVAMU-HEP-94-5

June 1994

\title{
On the Unitarity Triangles of the CKM Matrix
}

\author{
Dan-di WU中 \\ HEP, Box 355, Prairie View A\&M University, Prairie View, TX 77446, USA \\ and \\ Zhi-zhong XING' \\ Sektion Physik der Universität München, D-80333 Munich, Germany
}

\begin{abstract}
The unitarity triangles of the $3 \times 3$ Cabibbo-Kobayashi-Maskawa (CKM) matrix are studied in a systematic way. We show that the phases of the nine CKM rephasing invariants are indeed the outer angles of the six unitarity triangles and measurable in the $C P$-violating decay modes of $B_{d}$ and $B_{s}$ mesons. An economical notation system is introduced for describing properties of the unitarity triangles. To test unitarity of the CKM matrix we present some approximate but useful relations among the sides and angles of the unitarity triangles, which can be confronted with the accessible experiments of quark mixing and $C P$ violation.
\end{abstract}

\footnotetext{
${ }^{1}$ Supported in part by the U.S. Department of Energy under contract DE-FG05-92ER4072S

${ }^{2}$ Alexander-von-Humboldt Research Fellow
} 
In the standard electroweak model, the $3 \times 3$ Cabibbo-Kobayashi-Maskawa (CKM) matrix $V$ describes flavor mixing and $C P$ violation [1]. Unitarity is the only constraint, imposed by the model itself, upon $V$. Deviations from the minimal standard model may give rise to or fake as unitarity breaking. For example, unitarity of the $3 \times 3 \mathrm{CKM}$ matrix will not hold if there are fourth-family quarks or exotic charge $-1 / 3$ quarks which mix with the standard down-type quarks. It is therefore very important to stringently test unitarity of the CKM matrix. Recently, a lot of theoretical and experimental attention has been paid to this subject [2-8], in particular, to the six unitarity triangles. In Ref. [4], Aleksan et al have proved that four independent inner angles of the six triangles can provide a complete parametrization of the CKM matrix. Their results sharpen the importance of measuring $C P$ violation in $B$-meson decays so as to determine the entire quark mixing matrix $V$.

In this paper we shall make a systematic study of the unitarity triangles using the rephasing invariant quantities of the CKM matrix defined previously by one of the authors [9]. We show that the phases of the nine rephasing invariants are indeed the outer angles of the six unitarity triangles and directly related to the $C P$-violating asymmetries in some distinct decay modes of $B_{d}$ and $B_{s}$ mesons. To fully test unitarity of the standard model we give some useful relations among the sides and angles of the unitarity triangles, which can be confronted with the forthcoming experiments of quark mixing and $C P$ violation. It should be emphasized that our starting point of view differs from that in Ref. [4], nevertheless, some results of these two papers are in agreement with and supplementary to each other. In addition, we give a more economical notation scheme for the six unitarity triangles and present a practical way to check unitarity of the CKM matrix with the accessible experimental data. Recognizing that a few distinct and concise parametrization forms of the CKM matrix have been extensively applied to the studies of flavor mixing and $C P$ violation [6-8], the work done here is expected to provide a systematic approach to the same problem.

The unitarity triangles are defined by the following orthogonality relations:

$$
\begin{array}{ll}
\sum_{\alpha=d, s, b} V_{i \alpha} V_{j \alpha}^{*}=0, & (i<j) ; \\
\sum_{i=u, c, t} V_{i \alpha} V_{i \beta}^{*}=0, & (\alpha<\beta) .
\end{array}
$$

Here and hereafter, Latin subscripts run over the up-type quarks $u, c$, and $t$; while Greek ones run over the down-type quarks $d, s$, and $b$. It is known that there exist nine rephasing 
invariants for the $3 \times 3$ CKM matrix $V[9]$ :

$$
\Delta_{i \alpha} \equiv V_{j \beta} V_{k \gamma} V_{j \gamma}^{*} V_{k \beta}^{*}, \quad(i, j, k \text { and } \alpha, \beta, \gamma \text { co }- \text { cyclic })
$$

Here each $\Delta_{i \alpha}$ is a product of the four matrix elements in the $2 \times 2$ submatrix without $V_{i \alpha}$. It is crucial for such products that either two diagonal or skew diagonal elements are complex conjugated. The co-cyclic rule of the subscripts ensures a definite set of $\Delta_{i \alpha}$. In the subsequent discussions we shall imply co-cyclic permutation of the subscripts whenever three different Latin and (or) Greek subscripts simultaneously appear in a formula. Note that the phases of $\Delta_{i \alpha}$, denoted by

$$
\Phi_{i \alpha} \equiv \arg \Delta_{i \alpha}, \quad\left(-\pi<\Phi_{i \alpha} \leq \pi\right)
$$

are also invariant under quark phase redefinitions. In the complex plane, $\Phi_{i \alpha}$ is not only the relative angle of the two vectors $V_{j \beta} V_{j \gamma}^{*}$ and $V_{k \beta} V_{k \gamma}^{*}$ but also that of another two vectors $V_{j \beta} V_{k \beta}^{*}$ and $V_{j \gamma} V_{k \gamma}^{*}$. Thus $\Phi_{i \alpha}$ is indeed an outer angle shared by two different unitarity triangles (see Fig. 1). Unitarity of the $3 \times 3 \mathrm{CKM}$ matrix requires that all the nine quantities $\Delta_{i \alpha}$ have a common imaginary part [10], defined as

$$
J \equiv \operatorname{Im} \Delta_{i \alpha}=\left|\Delta_{i \alpha}\right| \sin \Phi_{i \alpha}
$$

which is a measure of $C P$ violation in the standard model. From Eqs. (3) and (4) one can observe that all nine $\Phi_{i \alpha}$ should have the same sign as $J$.

With Eqs. (2) and (3), it is straightforward to obtain the sum rules for $\Phi_{i \alpha}$, which are similar to the orthogonality relations given in Eq. (1):

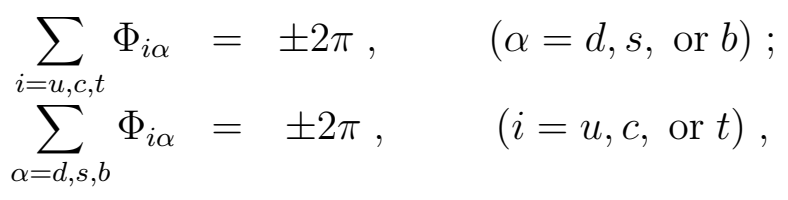

where the plus (minus) sign corresponds to $J>0(J<0)$. Since phase redefinitions of the quark fields cannot change the sign of $J$, the sign of $\Phi_{i \alpha}$ is definitely part of the information on the CKM unitarity. The current data on $\left|V_{u b} / V_{c b}\right|, x_{d}\left(B_{d}^{0}-\bar{B}_{d}^{0}\right.$ mixing), and $\epsilon_{K}$ (the $C P$-violating parameter in the kaon system) already indicate a positive $J$ [11]. This implies $\Phi_{i \alpha}>0$, corresponding to the anti-clockwise triangles in the complex plane (See Fig. 1). Subsequently we shall carry out the analysis under the condition of $J>0$. It is easy to follow a parallel analysis for the case of $J<0$. 
It proves convenient to use the capital form of those indices in the parentheses of Eq. (5) to name the unitarity triangles. For example, the $D$ triangle has three sides $S_{u D} \equiv$ $\left|V_{u s} V_{u b}^{*}\right|, S_{c D} \equiv\left|V_{c s} V_{c b}^{*}\right|$, and $S_{t D} \equiv\left|V_{t s} V_{t b}^{*}\right|$, which face three outer angles $\Phi_{u d}, \Phi_{c d}$, and $\Phi_{t d}$, respectively. Here the rule is that the CKM matrix elements with the subscript $d$ never appear in the $D$ triangle. In other words, the $D$ triangle can only be formed by the elements in the second and third columns of the CKM matrix. It should be noted that the six unitarity triangles have only nine different outer angles $\left(\Phi_{i \alpha}\right)$, although they have eighteen different sides $\left(\left|V_{j \beta} V_{j \gamma}^{*}\right|\right.$ and $\left.\left|V_{j \beta} V_{k \beta}^{*}\right|\right)$.

The inner angles of the unitarity triangles, denoted by $\omega_{i \alpha}$, satisfy the sum rules of the Euclidean geometry:

$$
\sum_{i=u, c, t} \omega_{i \alpha}=\sum_{\alpha=d, s, b} \omega_{i \alpha}=\pi
$$

They are related to the outer angles $\Phi_{i \alpha}$ through

$$
\omega_{i \alpha}=\pi \mp \Phi_{i \alpha}
$$

where the minus (plus) sign corresponds to $J>0(J<0)$. From the above relations we see that the roles of outer and inner angles are equivalent in discussing flavor mixing and $C P$ violation. For example, one can use $\Phi_{u s}, \Phi_{c s}$, and $\Phi_{t s}$ to describe the $S$ triangle, whose inner angles are commonly defined by $\alpha, \beta$, and $\gamma$ in the literature [12-14].

As to the magnitudes of the outer angles $\Phi_{i \alpha}$, Eq. (5) shows that the smaller $\left|\Delta_{i \alpha}\right|$, the larger $\sin \Phi_{i \alpha}$. In particular, we have

$$
\sin \Phi_{t b} \sim \lambda^{4}, \quad \sin \Phi_{u d} \sim \lambda^{2},
$$

where $\lambda^{2} \sim \frac{1}{20}$. The magnitudes of the remaining seven $\sin \Phi_{i \alpha}$ are of order $O(1)$.

It should be noted that one of the six relations in Eq. (5) is trivial. This implies that only four of the nine outer angles $\Phi_{i \alpha}$ are independent. It is easy to prove that such four independent $\Phi_{i \alpha}$ are enough to fully determine the magnitudes of the CKM matrix elements $\left|V_{i \alpha}\right|$. Indeed, we have the following relations between the sides and outer angles of the unitarity triangle $K$ (or $\Gamma)$ :

$$
\begin{aligned}
S_{K \alpha} \sin \Phi_{k \beta} & =S_{K \beta} \sin \Phi_{k \alpha}, \\
S_{i \Gamma} \sin \Phi_{j \gamma} & =S_{j \Gamma} \sin \Phi_{i \gamma} .
\end{aligned}
$$

\footnotetext{
${ }^{3}$ Analytically, $\omega_{i \alpha} \equiv\left|\arg \left(-\Delta_{i \alpha}^{*}\right)\right|$.
} 
As a result, all nine $\left|V_{i \alpha}\right|$ can be given in terms of $\Phi_{i \alpha}$, for example [15], from

$$
\frac{\left|V_{i \alpha}\right|^{2}}{\left|V_{i \beta}\right|^{2}}=\frac{S_{K \alpha} S_{I \beta} S_{J \alpha}}{S_{K \beta} S_{I \alpha} S_{J \beta}}=\frac{\sin \Phi_{k \alpha} \sin \Phi_{i \beta} \sin \Phi_{j \alpha}}{\sin \Phi_{k \beta} \sin \Phi_{i \alpha} \sin \Phi_{j \beta}}
$$

and the normalization condition $\sum_{i=u, c, t}\left|V_{i \alpha}\right|^{2}=1$. At this point, it is also worthwhile to give the relations between the sides and the cosines of the outer angles:

$$
\cos \Phi_{k \gamma}=\frac{S_{K \gamma}^{2}-S_{K \alpha}^{2}-S_{K \beta}^{2}}{2 S_{K \alpha} S_{K \beta}}=\frac{S_{k \Gamma}^{2}-S_{i \Gamma}^{2}-S_{j \Gamma}^{2}}{2 S_{i \Gamma} S_{j \Gamma}} .
$$

The cosines should be more sensitive to small alterations of the angles if their values are close to $\pi / 2$.

In practice, it will be convenient to choose $\Phi_{u s}, \Phi_{c s}, \Phi_{c d}$, and $\Phi_{u d}$ as a set of four independent parameters. These four phases are directly related to the observables of $C P$ violation in neutral $B$ decays [16], and therefore can be well determined in the near future. For illustration, we list in Table 1 some promising decay modes of $B_{d}$ and $B_{s}$ mesons, which can be used to probe the outer angles $\Phi_{i \alpha}$ in experiments.

We proceed with some discussions about testing unitarity of the CKM matrix. Due to various experimental limitations and theoretical uncertainties, a perfect test of the CKM unitarity is in reality impossible. Hence a practical or approximate check of the unitarity conditions is worth pursuing. According to Eq. (8),

$$
\Phi_{t b}=\Phi_{u d}=\pi
$$

is a good approximation up to $O\left(\lambda^{2}\right)$. Using Eq. (12) we obtain four simple sum rules for the outer angles $\Phi_{i \alpha}$ :

$$
\begin{aligned}
& \Phi_{t d}+\Phi_{t s}=\pi+O\left(\lambda^{4}\right), \\
& \Phi_{u b}+\Phi_{c b}=\pi+O\left(\lambda^{4}\right), \\
& \Phi_{u s}+\Phi_{u b}=\pi+O\left(\lambda^{2}\right), \\
& \Phi_{c d}+\Phi_{t d}=\pi+O\left(\lambda^{2}\right) .
\end{aligned}
$$

In a slightly different form, the above relations are expressed as

$$
\begin{aligned}
& \sin \Phi_{t d}=\sin \Phi_{t s}=\sin \Phi_{c d} \\
& \sin \Phi_{u b}=\sin \Phi_{c b}=\sin \Phi_{u s}
\end{aligned}
$$

which are valid up to $O\left(\lambda^{2}\right)$. From Eq. (12), some similar approximate relations may be obtained, up to $O\left(\lambda^{4}\right)$, for the sides of the unitarity triangles:

$$
\begin{array}{ll}
S_{U b}=S_{U s}, & S_{c D}=S_{t D} ; \\
S_{T d}=S_{T s}, & S_{c B}=S_{u B} .
\end{array}
$$


Clearly, all these approximate relations are experimentally accessible in the near future. Any evidence which conflicts with the above relations should be a signal of unitarity breaking.

For a complete test of unitarity, one has to check at least nine independent relations, in which at least one normalization condition is included and at least one outer (or inner) angle is involved. Fortunately, the six elements of the first two rows in the CKM matrix have already been measured and they satisfy the normalization conditions up to $O\left(\lambda^{4}\right)[5]$. Except for $S_{U b}, S_{U s}$, and $S_{t D}$, the other sides in Eq. (15) can now be determined very well. The relations $S_{T d}=S_{T s}$ and $S_{c B}=S_{u B}$ are consistent with the relevant data. No doubt, a precise determination of $\left|V_{u b}\right|$ is possible in further experiments of $B$-meson decays [17]. On the other hand, measurements of the top-quark lifetime will sharpen the value of $\left|V_{t b}\right|$, which is expected to be unity under the unitarity conditions [5]. The magnitudes of $V_{t d}$ and $V_{t s}$ are considerably difficult to be measured in direct decays of the top quark [18]. They may be determined indirectly from the forthcoming precise data on $B_{d}^{0}-\bar{B}_{d}^{0}$ and $B_{s}^{0}-\bar{B}_{s}^{0}$ mixings, respectively.

The problem is that one has not obtained any definite information on the angles of the unitarity triangles to check the relations in Eqs. (12) and (14). Progress is therefore dependent upon measuring $C P$ violation in nonleptonic $B$ decays, as illustrated in Table 1. In the literature, many approaches have been proposed to reliably extract the CKM phase from the expected large $C P$ asymmetries in the $B$-meson system [12-14, 16]. All these discussions are useful to determine the phases $\Phi_{i \alpha}$.

In summary, we have explored various relations among the outer and inner angles as well as the sides of the CKM unitarity triangles in a rephasing invariant way. The outer angles $\Phi_{i \alpha}$ are emphasized in describing flavor mixing and $C P$ violation within the standard model. To test unitarity of the $3 \times 3$ CKM matrix, we have presented some approximate but useful relations among the outer angles and sides, which are accessible to experiments in the near future.

One of us (Z.Z.X.) would like to thank Professor H. Fritzsch for his hospitality and encouragements. He is also indebted to the Alexander von Humboldt Foundation for its financial support. 


\section{References}

[1] N. Cabibbo, Phys. Rev. Lett. 10 (1963) 531; M. Kobayashi and T. Maskawa, Prog. Theor. Phys. 49 (1973) 552.

[2] J.D. Bjorken and I. Dunietz, Phys. Rev. D36 (1987) 2109; C. Jarlskog, in CP Violation, ed. C. Jarlskog (World Scientific, Singapore, 1988), p.1; I.I. Bigi, V.A. Khoze, N.G. Uraltsev, and A.I. Sanda, in the same book; I.I. Bigi and F. Gabbiani, Nucl. Phys. B352 (1991) 309.

[3] Y. Nir and H.R. Quinn, Phys. Rev. D42 (1990) 1473; Annu. Rev. Nucl. Part. Sci. 42 (1992) 211; I.I. Bigi, Preprint CERN-TH.7207/94 \& UND-HEP-94-BIG04 (1994); M. Kobayashi, Prog. Theor. Phys. 92 (1994) 287.

[4] R. Aleksan, B. Kayser, and D. London, Phys. Rev. Lett. 73 (1994) 18.

[5] For a review of experimental constraints on the CKM matrix and its unitarity, see: Particle Data Group, K. Hikasa, et al., Phys. Rev. D45-II (1992) III.65.

[6] L. Wolfenstein, Phys. Rev. Lett. 51 (1984) 1945.

[7] L.L. Chau and W.Y. Keung, Phys. Rev. Lett. 53 (1984) 1802.

[8] H. Fritzsch and J. Plankl, Phys. Rev. D35 (1987) 1732; H. Harari and M. Leurer, Phys. Lett. B181 (1986) 123; L. Maiani, Phys. Lett. B62 (1976) 183; M. Kobayashi and T. Maskawa, Ref. [1].

[9] D.D. Wu, Phys. Rev. D33 (1986) 860.

[10] L.L. Chau and W.Y. Keung, Ref. [7]; C. Jarlskog, Phys. Rev. Lett. 55 (1985) 1039; O.W. Greenberg, Phys. Rev. D32 (1985) 1841; D.D. Wu, Ref. [9].

[11] With the Wolfenstein parametrization of the CKM matrix [6], $J \approx A^{2} \lambda^{6} \eta$. All current data favor a positive $\eta$, which means $J>0$. The latest constraints on $\eta$ and other parameters can be found, e.g., in Ref. [17].

[12] I.I. Bigi, V.A. Khoze, N.G. Uraltsev, and A.I. Sanda, in CP Violation, ed. C. Jarlskog (World Scientific, Singapore, 1988), p.175; I.I. Bigi, Z. Phys. C51 (1991) 267. 
[13] P. Krawczyk, D. London, R.D. Peccei, and H. Steger, Nucl. Phys. B307 (1988) 19; C.O. Dib, I. Dunietz, F.J. Gilman, and Y. Nir, Phys. Rev. D41 (1990) 1522; and Ref. [3].

[14] M. Gronau and D. London, Phys. Lett. B253 (1991) 483; M. Gronau and D. Wyler, Phys. Lett. B265 (1991) 172; I. Dunietz, Phys. Lett. B270 (1991) 75; Z. Phys. C56 (1992) 129; D. Du and Z.Z. Xing, Phys. Rev. D47 (1993) 2825.

[15] Following a different approach, the similar results have been obtained in Ref. [4].

[16] H. Fritzsch, D.D. Wu and Z.Z. Xing, Phys. Lett. B328 (1994) 477; Z.Z. Xing, Phys. Rev. D50 (1994) R2957.

[17] A. Ali and D. London, Preprints CERN-TH.7248/94 \& UdeM-LPN-TH-94-197 (1994); A.J. Buras, Preprint MPI-PhT/94-25 \& TUM-T31-61/94 (1994).

[18] See, e.g., J.L. Rosner, Preprint EFI 90-63 (1990). 


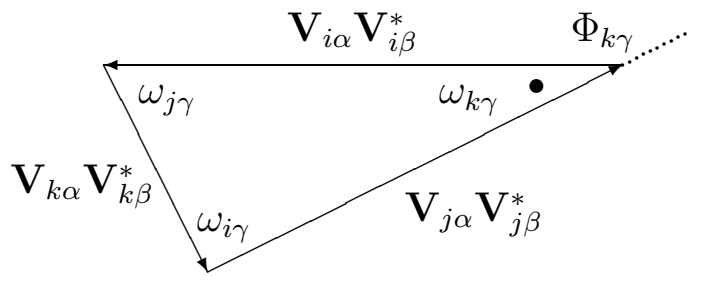

(Г)

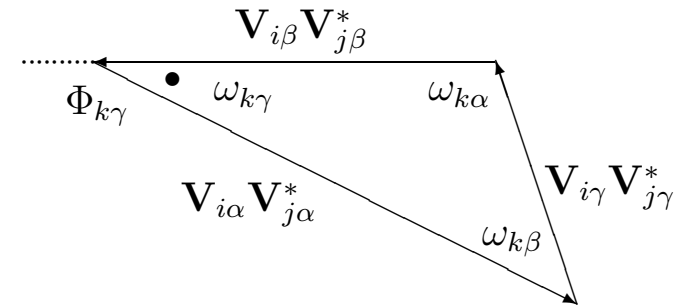

(K)

Figure 1: The CKM unitarity triangles $\Gamma(=D, S, B)$ and $K(=U, C, T)$ in the complex plane. A common outer angle $\Phi_{k \gamma}$ is shared by both triangles. Note the relation between the outer angle $\Phi_{k \gamma}$ and the inner angle $\omega_{k \gamma}$.

\begin{tabular}{l|l}
\hline \hline & \\
Promising decay modes & Measurable weak phases $\Phi_{i \alpha}$ \\
\hline & \\
$\stackrel{(-)}{B}_{d}^{0} \rightarrow J / \psi K_{S}, D^{+} D^{-}$ & $\sin 2 \Phi_{u s}$ \\
$\stackrel{(-)}{B}_{d}^{0} \rightarrow \pi^{+} \pi^{-}$ & $\sin 2 \Phi_{c s}$ \\
$\stackrel{(-)}{B}_{d}^{0} \rightarrow \stackrel{(-)}{D}^{(*) 0} K_{S}$ & $\sin \left(\Phi_{u s}+\Phi_{c d}-\Phi_{u b}\right)$ \\
$\stackrel{(-)}{B}_{d}^{0} \rightarrow D^{(*) \pm} \pi^{\mp}$ & $\sin \left(\Phi_{u s}-\Phi_{c s}\right)$ \\
& \\
$\stackrel{(-)}{B}_{s}^{0} \rightarrow J / \psi \phi, D_{s}^{+} D_{s}^{-}$ & $\sin 2 \Phi_{u d}$ \\
$\stackrel{(-)}{B}_{s}^{0} \rightarrow \rho^{0} K_{S}$ & $\sin 2\left(\Phi_{c d}-\Phi_{t b}\right)$ \\
$\stackrel{(-)}{B}_{s}^{0} \rightarrow \stackrel{(-)}{D}^{(*) 0} \phi$ & $\sin \left(\Phi_{c d}-\Phi_{u d}\right)$ \\
$\stackrel{(-)}{B}_{s}^{0} \rightarrow \stackrel{(-)}{D}^{(*) 0} K_{S}$ & $\sin \left(\Phi_{u b}-\Phi_{u d}-\Phi_{c s}\right)$ \\
\hline \hline
\end{tabular}

Table 1: Some promising decay modes of $B_{d}$ and $B_{s}$ mesons and specific rephasing invariant weak phases $\Phi_{i \alpha}$, which are determinable in their $C P$ asymmetries. 